\title{
Early social deprivation negatively affects social skill acquisition in chimpanzees (Pan troglodytes)
}

\author{
Edwin J. C. van Leeuwen · Innocent Chitalu Mulenga • \\ Diana Lisensky Chidester
}

Received: 15 April 2013/Revised: 1 August 2013/Accepted: 7 August 2013/Published online: 17 August 2013

(C) Springer-Verlag Berlin Heidelberg 2013

\begin{abstract}
In a highly social species like chimpanzees, the process by which individuals become attuned to their social environment may be of vital importance to their chances of survival. Typically, this socialization process, defined by all acquisition experiences and fine-tuning efforts of social interaction patterns during ontogeny, occurs in large part through parental investment. In this study, we investigated whether maternal presence enhances the socialization process in chimpanzees by comparing the social interactions of orphaned and mother-reared individuals at the Chimfunshi Wildlife Orphanage Trust in Zambia. As response variables, we selected social interactions during which an elaborate level of fine-tuning is assumed to be necessary for sustaining the interaction and preventing escalation: social play. Comparing orphaned $(n=8)$ to sex- and age-matched mother-reared juvenile chimpanzees $(n=9)$, we hypothesized that the orphaned juveniles would play less frequently than the mother-reared and would be less equipped for fine-tuning social play (which we assayed by rates of aggression) because of the lack of a safe and facilitating social environment provided by the mother. First, contrary to our hypothesis, results showed
\end{abstract}

Electronic supplementary material The online version of this article (doi:10.1007/s10071-013-0672-5) contains supplementary material, which is available to authorized users.

E. J. C. van Leeuwen ( $\square)$

Max Planck Institute for Psycholinguistics, PO Box 310, 6500

AH Nijmegen, The Netherlands

e-mail: edwin.vanleeuwen@mpi.nl

E. J. C. van Leeuwen - I. C. Mulenga - D. L. Chidester Chimfunshi Wildlife Orphanage Trust, Chingola, Zambia e-mail: chimfunshiwildlife@iwayafrica.com

D. L. Chidester

e-mail: lisensky.chidester@gmail.com that the orphaned juveniles engaged in social play more frequently than the mother-reared juveniles, although for significantly shorter amounts of time. Second, in support of our hypothesis, results showed that social play of the orphaned juveniles more often resulted in aggression than social play of the mother-reared juveniles. In conjunction, these results may indicate that, just like in humans, chimpanzee mothers provide their offspring with adequate social skills that might be of pivotal importance for future challenges like successful group-living and securing competitive fitness advantages.

Keywords Socialization - Chimpanzees - Maternal deprivation - Social competence $\cdot$ Maternal care . Social play

\section{Introduction}

Socialization refers to the process during which individuals of a social species acquire and develop skills that are appropriate for adequate group-living, hence maintaining or increasing fitness (Munkenbeck and Mitchell 1974; Rapaport and Brown 2008). In primates, typically, a suite of behaviours must be learned by infants and juveniles before reaching mature independence, including foraging (e.g. locations and tool use), recognizing dominance indicators, reproductive behaviours, nest building, mothering and more general social behaviours like affiliative and submissive behaviours (Mason 1967; Poirier 1977; Russon 1990; Watts and Pusey 2002). Mothers play an important role in the socialization of primates (Hrdy 1999; Lonsdorf and Ross 2012), where early studies have shown that the absence of mothers during sensitive periods in infancy can have detrimental effects on the physical and social 
development of individuals, even resulting in the near absence of future reproductive success (Harlow and Harlow 1962; Novak and Sackett 2006).

Chimpanzees are a social species exhibiting interactive behaviours that require a high level of coordination for their successful execution, like cooperative hunting, coalitionary aggression and boundary patrolling [e.g. (Boesch and Boesch 1989; Mitani 2009a)]. While the adequate execution of these behaviours may confer substantial fitness benefits, chimpanzees need a long period of socialization in order to acquire and fine-tune their socially complex behaviour [e.g. (Lonsdorf and Ross 2012; Mason 1986; Poirier 1972, 1977; Watts and Pusey 2002)]. Typically, chimpanzees are very vulnerable from birth and spend the majority of their first years dependent on their mothers (Goodall 1986). During this period, chimpanzees obtain an important amount of knowledge on the speciestypical, and possibly group-specific, repertoire of individual and social behaviours (Goodall 1986; Rapaport and Brown 2008), where the presence of the mother seems to elicit skill acquisition in the infants (Biro et al. 2003; Lonsdorf 2013) and even active teaching by chimpanzee mothers has been observed in the wild (Boesch 1991).

In this study, we aimed to investigate the extent to which mother-rearing affects the adequacy of chimpanzees' social behaviour in later life by comparing the characteristics of social play between orphaned and mother-reared individuals. Play is often considered practice of skills that prepare individuals for future events (Burghardt 2005; Spinka et al. 2001), and as an important means by which individuals establish and maintain social relationships in general (Fagen 1993; Palagi 2006) and acquire information on group members' behavioural tendencies in particular (Ciani et al. 2012; Pellis and Pellis 2009). More importantly for the purpose of this study, social play entails interactive behaviour that requires an elaborate level of fine-tuning for its execution [e.g. (Bekoff and Allen 1998; Palagi et al. 2007)], and comprises a context in which individuals, upon inadequate fine-tuning with their play partner, risk severe injuries or even physical exclusion from their social group (Bekoff and Pierce 2009; Burghardt 2005). Moreover, social play is considered to be one of the most complex types of social communication for it entails an ongoing exchange of social information, which requires adequate sending and receiving of signals (Burghardt 2005; Pellis and Pellis 2009). In other words, social play may be both an assay for general social skill proficiency [also see (Lewis 2000)] and may influence the fitness potential of individuals directly by affecting factors like physical condition and social status [also see (Palagi 2011)]. In this study, first, we compared the frequencies and durations of social play behaviour between orphaned and mother-reared juvenile chimpanzees to assess the proficiency of the chimpanzees' social skills. Here, we hypothesized that the mother-reared chimpanzees would engage in social play more frequently and for longer continuous durations than their orphaned counterparts for the reason that we expected the mother-reared chimpanzees to be more socially attuned, i.e., more proficient at soliciting and sustaining social play, owing to their typical social development [e.g. (Maki et al. 1993; Russell et al. 2011)]. Second, we investigated the adequacy of the chimpanzees' social skills by analysing the social play outcomes in terms of aggression. Here, we hypothesized that the orphaned chimpanzees would start fighting during social play more frequently than their mother-reared counterparts for the reason that we expected the orphaned chimpanzees to be less able to adequately communicate (both signalling and perceiving) that their employed behaviour is not aggressively, but playfully intended [e.g. (Bekoff 2004; van Hooff 1973)]. Under the parsimonious assumption that positive social relationships and group-living confer fitness benefits to social animals including chimpanzees [e.g. (Langergraber et al. 2013; Silk et al. 2003; Vanschaik and Vanhooff 1983)], both the proficiency and adequacy of social behaviour may (in)directly affect chimpanzees' fitness potential [also see (Taborsky and Oliveira 2012) for a general appraisal of the impact of social competence on Darwinian fitness].

\section{Materials and methods}

\section{Subjects}

All chimpanzees that were observed for the current study lived at the Chimfunshi Wildlife Orphanage Trust (CWOT), a sanctuary that houses more than a hundred chimpanzees under close to natural conditions in the northwestern part of Zambia (for details, see van Leeuwen et al. 2012). The group of orphaned juveniles consisted of 11 individuals of which 8 individuals (4 females, 4 males; $M_{\text {age }} 7.6$ years; age range 4-9 years) were selected for this study (1 individual was too young to be socially active and was still regularly hand-reared by humans; the 2 others were only sometimes integrated in the group because of management reasons). All orphans were wild-born and were brought into CWOT at the age of 3 years or younger. The available records showed that all orphans had been taken away from their mother within their first year of life (S. Siddle \& I. Mulenga, pers.comm. 2012, founder and current manager of CWOT, respectively). At CWOT, the orphans roamed freely outside in a 3.24 ha enclosure from 8:00 am to 12:00 pm, after which they were kept inside for the remainder of the day. The enclosure consisted of wild Miombo woodland, which comprises many opportunities 
for the chimpanzees to forage, climb and build nests [see (Ron and Mcgrew 1988)]. The indoor facility consisted of four connected cages through which the chimpanzees could roam freely (approx. $80 \mathrm{~m}^{2}$ ). Furthermore, the orphans were being taken on so-called bushwalks irregularly, which comprised a 1-2 h interaction with human visitors. Data were only collected in the absence of visitors, but since interaction with visitors may affect the subsequent behaviour of the orphaned juveniles, we investigated this influence in more detail by comparing play behaviour between bushwalk days and non-bushwalk days.

The group of mother-reared juveniles consisted of 9 individuals ( 5 females, 4 males; $M_{\text {age }} 7.3$ years; age range 4-8 years), belonging to a social group of 47 individuals (including their mothers) at the start of the study. The mother-reared chimpanzees lived in a 64.76 ha enclosure (consisting of similar Miombo woodland as the enclosure of the orphaned chimpanzees), where they roamed freely throughout the day, except for midday feeding in an indoor holding-facility between 11:30 and 13:30. No bushwalks were undertaken with the chimpanzees from this group.

\section{Behavioural coding}

Behaviour was coded according to the ethogram of the Chimpanzee and Human Communication Institute (2000; see Online Resource 1 for the specific ethogram used in this study). Social play behaviour was defined by two or more subjects interactively engaging in "play", where only bouts between peers were included for further analysis (i.e. only bouts between orphaned juveniles in the orphaned group and bouts between mother-reared juveniles in the mother-reared group were included). In more detail, social play comprised any non-aggressive interactions between two or more individuals that included one or a combination of the following behaviours: tickling, wrestling, biting, sparring (hitting and pushing), chasing, butting, kicking, dragging, finger wrestling, pinching, rubbing, thrusting, play face, play walk and laughing. In order to investigate play initiation rates and the perpetuation of play bouts, play duration was categorized a priori in mutually exclusive timescales comprising 0-5-, 6-10-, 11-19-, 20-29-s, 30 s1-min, 1-2-, 2-3-, 3-4-, 4-5-, and >5-min intervals, where a play bout started with the first playful interaction between two subjects and ended when there was no playful behaviour from any of the involved partners for $\geq 5 \mathrm{~s}$. To assess durations of social play behaviour, the average of the time-interval boundaries was taken (e.g. 3 social play bouts in the 20-29-s interval would yield a duration of 3 times $((20+29) / 2)=73.5 \mathrm{~s})$. Play outcomes were recorded as aggressive when at least one of the interacting subjects would engage in agonistic behaviour. In more detail, agonistic behaviour comprised any aggressive interactions between two or more individuals that included one or a combination of the following behaviours: poking, kicking, biting, hitting, throwing an object at another, or hitting another with an object. Furthermore, to be able to better distinguish between playful and aggressive behaviour, we only coded "aggression" when one or both of the play partners started screaming. To control for non-independence, only one aggressive play outcome was included for analysis per focal follow. All statistical tests are two-tailed unless specified otherwise. We applied the Holm-Bonferroni method to control the family-wise error rate (Holm 1979).

\section{Observations}

We used a 15-min focal sampling technique (Martin and Bateson 1994), where individuals were randomly selected as focal individuals based on visibility and location of the observer (as they walked along the fence line in order to find the chimpanzees). Random selection was done by a random name-generation procedure without replacement, but when the designated individual could not be found for $10 \mathrm{~min}$, the next name was generated. Three observers live coded a total of 92 focal hours with an average of $5.4 \mathrm{~h}$ per subject (range 3.7-7.3 and 2.9-9.7 $\mathrm{h}$ for the orphaned and mother-reared juveniles, respectively). The observation period lasted from the 15 th of June 2007 until the 15 th of August 2007, where observations on both groups were only done between 8:00 am and 12:00 pm in order to control for daytime differences (i.e. the orphaned chimpanzees were only outside between 8:00 am and 12:00 pm). Of the data collected on the orphaned juveniles, 14.9 focal hours were obtained on "bushwalk days" (days on which the chimpanzees physically interacted with human visitors) and 30.2 h on "non-bushwalk days". Since we neither found a significant difference between bushwalk days and nonbushwalk days in the frequency of social play (Wilcoxon test: $p=0.93$ ) nor the frequency by which social play resulted in aggression (Wilcoxon test: $p=0.61$ ), we pooled the data and report them accordingly. Inter-observer reliability was established prior to data collection by simultaneously, yet independently live coding $2.5 \mathrm{~h}$ of focal follow data. The calculation was based on comparing the rates of agreement across 7 behavioural categories (social play, lone play, forage, rest, explore, aggression and grooming: see Chimpanzee and Human Communication Institute 2000) between the three observers, where they had coded the behaviour of the focal individual every $15 \mathrm{~s}$ as indicated by an audible signal from a stopwatch. The observations for the inter-observer reliability test were done during the 2 -h window preceding feeding time for the reason that during this window, the chimpanzees are most active and the reliability assessment thus maximized its 
purpose by allowing calculation on a relative diverse set of observations [observer $1 \& 2$ : Cohen's $K=0.84$; observer 1 \& 3: Cohen's $K=0.85$; observer 2 \& 3: Cohen's $K=0.80$; (Cohen 1960)]. Moreover, two out of the three observers conducted focal follow observations in both chimpanzee groups, which further increased the validity of our combined observations.

\section{Results}

Social play proficiency: frequencies and duration

Overall, the orphaned chimpanzees engaged in social play behaviour more frequently than the mother-reared chimpanzees (Mann-Whitney test: $U=4.0, \quad Z=-3.08$, $N_{1}=8, \quad N_{2}=9, p=0.001$; mean \pm SD: 5.85 bouts/ $\mathrm{h} \pm 0.44,1.96$ bouts $/ \mathrm{h} \pm 0.23$, respectively; see Online Resource 2 for a table with the frequencies across the two groups broken down into the different social play intervals). On the other hand, we found no difference in the proportion of time that the orphaned and mother-reared chimpanzees engaged in social play behaviour $(U=41.0$, $Z=0.48, \quad N_{1}=8, \quad N_{2}=9, \quad p=0.67 ; \quad$ mean $\pm \mathrm{SD}$ : $13.10 \% \pm 7.96,15.61 \% \pm 10.39$, respectively). Closer inspection of the data revealed that the orphaned chimpanzees spent more time engaging in relatively short social play bouts, while the mother-reared chimpanzees spent more time engaging in relatively long social play bouts (see Fig. 1). In more detail, comparing the two study groups, the orphaned chimpanzees engaged significantly longer in the 5-, 6-10-, and 11-19-s social play intervals $(p<0.01$, $p<0.01$, and $p=0.012$, respectively), while the motherreared chimpanzees seemed to engage significantly longer in the 2-3-min and 3-4-min intervals $(p=0.030$, and $p=0.059$, respectively; both moderate trends when Holm-Bonferroni corrected).

\section{Social play adequacy: aggressive outcomes}

The social play bouts of the orphaned chimpanzees resulted significantly more often in aggression than the social play bouts of the mother-reared chimpanzees $(U=7.0, Z=$ $-2.83, N_{1}=8, N_{2}=9, p=0.004$; see Fig. 2). This result was robust in the sense that the same pattern was found after the exclusion of the most extreme data point (outlier) in the orphaned chimpanzees group $(19.1 \%=$ mean $+2.39 \mathrm{SD})$, indicating that the group difference was not due to one individual $\left(U=7.0, Z=-2.63, N_{1}=7\right.$, $N_{2}=9, p=0.008$; see Fig. 2). Moreover, each orphaned chimpanzee was engaged in a social play bout that turned into aggression at least once with at least 3 different play partners (range 3-6), further indicating that the presented result reflects a group-level effect rather than an effect caused by individual differences (only 4 out of 9 motherreared chimpanzees were observed to be engaged in social play turning into aggression; 3 subjects with only one partner and 1 subject with 2 different partners).

\section{Discussion}

In this study, we opportunistically investigated whether the lack of maternal presence throughout infancy and juvenility affected chimpanzees' social skills later in life by comparing social play behaviour of orphaned juvenile chimpanzees to that of mother-reared juvenile chimpanzees. Orphaned chimpanzees were found to engage in social play more frequently than their age- and sex-matched mother-reared counterparts. However, their social play bouts were relatively short in comparison with the social play bouts of the mother-reared chimpanzees, indicating that although the orphaned chimpanzees were motivated to engage in social play, they were less capable of sustaining interactive play bouts. Moreover, the likelihood with which social play bouts turned into aggression was more than five times higher in the orphaned chimpanzees than the mother-reared chimpanzees. Corroborating previous reports [e.g. (Kalcher-Sommersguter et al. 2011; Maki et al. 1993; Russon 1990)], these data may imply that chimpanzees learn adequate social skills from their mothers throughout infancy and juvenility; skills that enhance the quality of interactions with conspecifics in later life, which, in a highly socially dependent species like chimpanzees may be of crucial importance for successful group-living or even immediate survival (Bekoff and Pierce 2009; Taborsky and Oliveira 2012).

The importance of early social experiences-and maternal care in particular-for offspring social development has been demonstrated in a diverse range of taxa [e.g. primates: (Rapaport and Brown 2008); rats: (Levy et al. 2003); fish: (Taborsky et al. 2012); birds: (White et al. 2010)]. Typically, animals that grow up in a socially dynamic environment or receive (adequate) maternal care throughout infancy and juvenility are more capable of optimising social behaviour later in life than their socially deprived counterparts, which can provide substantial fitness advantages (Taborsky and Oliveira 2012). In the current study, the social play behaviour of maternally deprived chimpanzees was characterized by a relatively impaired capacity to sustain playful interactions and prevent these interactions from turning into aggression. Where the former may indicate that chimpanzees that have missed an important part of their natural socialization process are less capable of establishing and maintaining close social bonds, the latter shows that lessons learned from mothers 


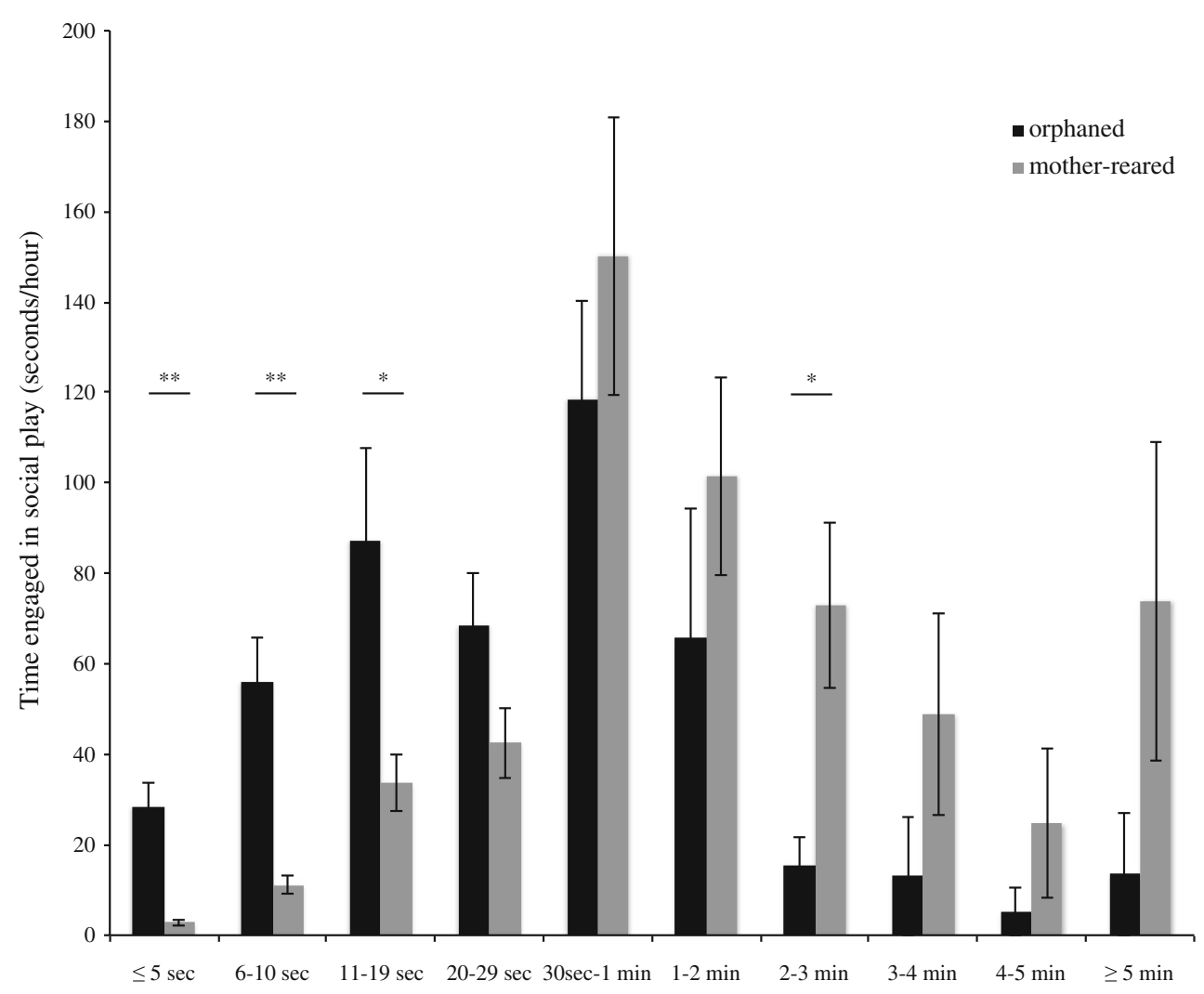

Fig. 1 Mean $( \pm$ s.e.m) time $(\mathrm{s} / \mathrm{h})$ that the orphaned (black bars) and mother-reared chimpanzees (grey bars) engaged in social play bouts of 10 increasingly long time intervals ( $x$ axis). One asterisk indicates

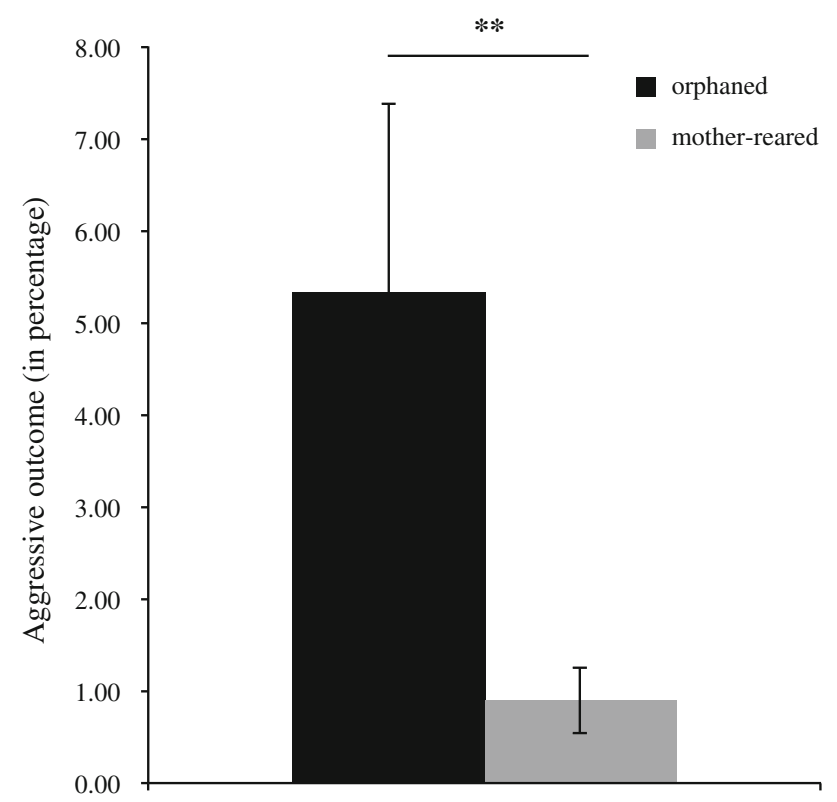

Fig. 2 Mean $( \pm$ s.e.m) percentage of social play bouts that ended in aggression for the orphaned $(5.34 \pm 2.03)$ and mother-reared chimpanzees $(0.90 \pm 0.36)$. Corrected mean $( \pm$ s.e.m) for the orphaned chimpanzees after removal of the outlier is $3.37 \pm 0.61 \%$. Asterisks indicate a significant difference at the 0.01 alpha level a significant difference at the 0.05 alpha level, and two asterisks indicate a significant difference at the 0.01 alpha level

(or being in the presence thereof) may actually safeguard chimpanzees from the physical and social risk inherent to aggressive encounters. A similar finding was reported in a recent bonobo study (Clay and de Waal 2013) focusing on the socially important dynamics of reconciliation and consolation (Aureli and de Waal 2000). Here, it was found that compared to their juvenile mother-reared counterparts, orphaned bonobos were less actively engaged in both reconciliation and consolation (Clay and de Waal 2013), which point towards a similar negative link between early social deprivation and the development of socially attuned behaviour as the findings of the present study.

An alternative explanation for the differences in social play behaviour between the orphaned and mother-reared chimpanzees may be that the mother-reared chimpanzees were still monitored and looked after by their mothers. Chimpanzee mothers are known to be protective of their offspring, even post-weaning (Goodall 1986; Nishida 2012). In this study, the chimpanzee mothers may have prevented escalation of their offspring's social play by intervening in increasingly intensive play bouts. This explanation, however, seems unlikely for the reason that the social play bouts of the mother-reared chimpanzees were typically longer than those of the orphaned 
chimpanzees. If mothers were to end social play bouts of their offspring, it would be expected to find shorter play bouts in mother-reared chimpanzees, unless there were additional factors compromising the duration of social play bouts in orphaned chimpanzees. One of these factors may have been the relative inability of orphaned chimpanzees to communicate the meta-information "this is only play" [e.g. see (Bekoff 2004; Burghardt 2005)]. Social animals use communicative signals during playful interactions, like facial expressions and vocalizations, to convey the message that whatever they are doing to the interaction partner is play, not aggression. In the case where the nature of the performed behaviour is difficult to determine by its physical appearance (i.e. play and aggression are comprised of similar behavioural elements, like hitting and biting), it is important for the play partners to communicate about the intentions of the ongoing, interactive behaviour (Bekoff and Byers 1998). In chimpanzees, the open-mouth face and exaggerated panting ("laughter") have been characterized as play signals (van Hooff 1972), even to the extent of producing a distinct laughter-type for communicating the willingness to continue playing, as contrasted to spontaneous laughter (Davila-Ross et al. 2011). Moreover, the intensity by which these signals are being communicated has been linked to the presence of the play partners' mothers (Flack et al. 2004). In the current study, the mother-reared chimpanzees may have been play-signalling more intensely than their orphaned counterparts because of the presence of their mothers. Albeit interesting to scrutinize in a follow-up study, this explanatory variable would still point to the fact that the mother-reared chimpanzees were more competent at coordinating intense social interactions than the orphaned chimpanzees. Alternatively, orphaned chimpanzees might have been less adequate in the timing of their play signals. For instance, Spijkerman et al. (1996) found that even though chimpanzees that were raised in peer groups used play faces equally or even more frequently during social play than chimpanzees that were living in a semi-natural zoo environment (in groups comprising family units and chimpanzees from all age classes), the peer group-raised chimpanzees used play faces less frequently when they were engaged in (playful) gnawing, which arguably comprises a behaviour in need of metainformation conveying its modality: e.g., "this is playfully intended, not aggressively" (Bekoff and Allen 1998). Future research might reveal valuable insights regarding the mechanism underlying the presented differences between the social play interactions of orphaned and mother-reared chimpanzees by focusing specifically on play-signalling during ambiguous (playful) behaviours, like gnawing, biting and hitting; especially in light of the intriguing finding that chimpanzees do not only produce distinct laughter types during social play, but that these expressions seem to emerge from socialization processes (Davila-Ross et al. 2011).

It is important to note that the orphaned chimpanzees were not only deprived of maternal care throughout infancy and juvenility, but that they have also remained deprived of any typically socialized role-model throughout the rest of their development. Knowing that chimpanzees typically form social bonds with a multitude of group members [e.g. see (Langergraber et al. 2009; Mitani 2009b)] and that they are proficient at socially acquiring social behaviour [e.g. (Lonsdorf 2013; van Leeuwen et al. 2012)], it would be interesting to find out to what extent the orphaned chimpanzees could adjust the adequacy of their social (play) behaviour in the presence of more socially attuned individuals. Furthermore, the presented results should be taken with caution, since group comparisons are inevitably prone to subtle, yet possibly meaningful differences. For instance, the difference in enclosure size and time spent in the indoor holding facilities may affect the social behaviour of the individuals. In this case, the former concern may be dismissed on the basis of the fact that even the smallest enclosure was sufficiently large to avoid social contact if preferred. The latter concern may be minimized by the fact that the indoor facility of the orphaned chimpanzees consisted of several connected spaces, where the chimpanzees would move through freely. Moreover, the playful interactions that turned into aggression were normally distributed across the time that the orphaned chimpanzees were outside (i.e. not clustered in the time immediately following release from their indoor holding spaces).

Finally, it might have been the case that early separation trauma intrinsically caused the orphaned chimpanzees to become less socially attuned, irrespective of the subsequent absence of maternal care. In line with this reasoning are the recent findings that chimpanzees can develop (signs of) mood and anxiety disorders after suffering traumatic experiences (Ferdowsian et al. 2011). Weighing these alternative explanations, however, we would argue that the deprivation of maternal care would be a more likely explanation of the presented results than the early trauma suffered by the orphaned chimpanzees. The typical symptoms that humans [and chimpanzees: (Ferdowsian et al. 2011)] develop after traumatic experiences are of an internalizing nature, i.e., symptoms that are characterized by social withdrawal rather than social engagement and are contrasted to externalizing symptoms like aggression (American Psychiatric Association 2000; Ferdowsian et al. 2011). The findings of the present study show the opposite pattern: the orphaned chimpanzees were both socially engaged (as reflected by the high frequency by which they initiated social play) and rather externally than internally oriented (as reflected by the aggressive outcomes of their social play bouts). Moreover, while the explored link 
between (early) trauma and resultant symptomatology warrants further investigation, the substantial research indicating that mothers are crucially important for a typical (social) development in chimpanzees plausibly grounds the tenet of our conclusions [e.g. (Goodall 1986; Lonsdorf and Ross 2012; Nishida 2012)].

In sum, the data of the present study suggest that maternal care (or at least the presence of socially attuned individuals) is essential for the development of social skills in chimpanzees. While these data underline the importance of the mother-infant bond in chimpanzees, this study further indicates that chimpanzees are not innately predisposed towards optimal (social) behaviour, nor that mere individual learning will sufficiently shape their behavioural repertoire: much like humans, chimpanzees seem to need social role models in order to be able to navigate their complex social lives.

Acknowledgment The authors would like to thank the Zambia Wildlife Authority for facilitating our research in Zambia and the management of the Chimfunshi Wildlife Orphanage Trust for hosting this project within their sanctuary. Many thanks go out to the chimpanzee keepers \& CWOT researchers (Patrick Chambatu, Felix Chinyama, Chrispin Kapango, Joseph Kasongo, Mumba Kawele, John Kayuya, Goodson Muletele, Thomson Mbilishi and Patrick Mwika) and to Katrina Seville for assisting in the data collection. We are grateful to Sheila Siddle (co-founder of CWOT) and to Katherine Cronin, Becky Koomen and two anonymous reviewers for helpful comments on an earlier draft. This work was supported by the Chimfunshi Wildlife Orphanage Trust.

\section{References}

American Psychiatric Association (2000) Diagnostic and statistical manual of mental disorders, 4th edn. American Psychiatric Association, Washington, DC

Aureli F, de Waal FBM (2000) Natural conflict resolution. University of California Press, Berkeley

Bekoff M (2004) Wild justice and fair play: cooperation, forgiveness, and morality in animals. Biol Philos 19(4):489-520. doi:10. 1007/sBIPH-004-0539-x

Bekoff M, Allen C (1998) Intentional communication and social play: how and why animals negotiate and agree to play. In: Bekoff $\mathrm{M}$, Byers B (eds) Animal play-evolutionary, comparative, and ecological perspectives. Cambridge University Press, Cambridge, pp 97-114

Bekoff M, Byers B (1998) Animal play: evolutionary, comparative, and ecological perspectives. Cambridge University Press, Cambridge

Bekoff M, Pierce J (2009) Wild justice: the moral lives of animals. Cambridge University Press, Cambridge

Biro D, Inoue-Nakamura N, Tonooka R, Yamakoshi G, Sousa C, Matsuzawa T (2003) Cultural innovation and transmission of tool use in wild chimpanzees: evidence from field experiments. Anim Cogn 6(4):213-223. doi:10.1007/S10071-003-0183-X

Boesch C (1991) Teaching among wild chimpanzees. Anim Behav 41:530-532. doi:10.1016/s0003-3472(05)80857-7

Boesch C, Boesch H (1989) Hunting behavior of wild chimpanzees in the Tai-National-Park. Am J Phys Anthropol 78(4):547-573. doi:10.1002/Ajpa.1330780410
Burghardt GM (2005) Genesis of animal play: testing the limits. MIT Press, Cambridge

Chimpanzee and Human Communication Institute (2000) Taxonomy of chimpanzee behaviors. Central Washington University, Ellensburg

Ciani F, Dall'Olio S, Stanyon R, Palagi E (2012) Social tolerance and adult play in macaque societies: a comparison with different human cultures. Anim Behav 84(6):1313-1322. doi:10.1016/j. anbehav.2012.09.002

Clay Z, de Waal FBM (2013) Bonobos respond to distress in others: consolation across the age spectrum. Plos One 8(1). doi:10.1371/ journal.pone.0055206

Cohen J (1960) A coefficient of agreement for nominal scales. Educ Psychol Meas 20(1):37-46

Davila-Ross M, Allcock B, Thomas C, Bard KA (2011) Aping expressions? Chimpanzees produce distinct laugh types when responding to laughter of others. Emotion 11(5):1013-1020. doi:10.1037/A0022594

Fagen R (1993) Primate juveniles and primate play. Juvenile primates: life history, development, and behavior. Oxford University Press, New York

Ferdowsian HR, Durham DL, Kimwele C, Kranendonk G, Otali E, Akugizibwe T, Mulcahy JB, Ajarova L, Johnson CM (2011) Signs of mood and anxiety disorders in chimpanzees. Plos One 6(6). doi:10.1371/journal.pone.0019855

Flack JC, Jeannotte LA, de Waal FBM (2004) Play signaling and the perception of social rules by juvenile chimpanzees (Pan troglodytes). J Comp Psychol 118(2):149-159. doi:10.1037/ 0735-7036.118.2.149

Goodall J (1986) The chimpanzees of Gombe: patterns of behavior. Harvard University Press, Boston

Harlow HF, Harlow MK (1962) The effect of rearing conditions on behavior. Bull Menninger Clin 26(5):213-224

Holm S (1979) A simple sequentially rejective multiple test procedure. Scand J Stat 6(2):65-70

Hrdy SB (1999) Mother nature: a history of mothers, infants, and natural selection. Pantheon Books, New York

Kalcher-Sommersguter E, Preuschoft S, Crailsheim K, Franz C (2011) Social competence of adult chimpanzees (Pan troglodytes) with severe deprivation history: I. An individual approach. Dev Psychol 47:77-90. doi:10.1037/a0024353

Langergraber K, Mitani J, Vigilant L (2009) Kinship and social bonds in female chimpanzees (Pan troglodytes). Am J Primatol 71(10):840-851. doi:10.1002/ajp.20711

Langergraber K, Mitani JC, Watts DP, Vigilant L (2013) Malefemale socio-spatial relationships and reproduction in wild chimpanzees. Behav Ecol Sociobiol. doi:10.1007/s00265-0131509-6

Levy F, Melo AI, Galef G, Madden M, Fleming AS (2003) Complete maternal deprivation affects social but not spatial learning in adult rats. Dev Psychobiol 43(3):177-191. doi:10.1002/dev. 10131

Lewis KP (2000) A comparative study of primate play behaviour: implications for the study of cognition. Folia Primatol 71(6):417-421. doi:10.1159/000052740

Lonsdorf EV (2013) The role of mothers in the development of tooluse in chimpanzees. In: Clancy KBH, Hinde K, Rutherford JN (eds) Building babies: primate development in proximate and ultimate perspective. Developments in primatology: progress and prospects. Springer, New York, pp 303-318

Lonsdorf A, Ross SR (2012) Socialization and development of behavior. In: Mitani J, Kappeler P, Palombit R, Silk JB (eds) Evolution of primate societies. The University of Chicago Press, Chicago, pp 245-268

Maki S, Fritz J, England N (1993) An assessment of early differential rearing conditions on later behavioral-development in captive 
chimpanzees. Infant Behav Dev 16(3):373-381. doi:10.1016/ 0163-6383(93)80042-7

Martin P, Bateson P (1994) Measuring behaviour: an introductory guide, 2nd edn. Cambridge University Press, Cambridge

Mason WA (1967) Motivational aspects of social responsiveness in young chimpanzees. Early behavior: comparative and developmental approaches. John Wiley \& Sons, New York

Mason WA (1986) Can primate political traits be identified? Primate ontogeny, cognition and social behavior. Cambridge University Press, Cambridge

Mitani JC (2009a) Cooperation and competition in chimpanzees: current understanding and future challenges. Evol Anthropol 18(5):215-227. doi:10.1002/Evan.20229

Mitani JC (2009b) Male chimpanzees form enduring and equitable social bonds. Anim Behav 77(3):633-640. doi:10.1016/j. anbehav.2008.11.021

Munkenbeck D, Mitchell G (1974) Infant socialization in primates. J Hum Evol 3(6):563-574

Nishida T (2012) Chimpanzees of the lakeshore: natural history and culture at Mahale. Cambridge University Press, Cambridge

Novak MA, Sackett G (2006) The effects of rearing experiences: the early years. Nursery rearing of nonhuman primates in the $21 \mathrm{st}$ century. Springer, Chicago

Palagi E (2006) Social play in bonobos (Pan paniscus) and chimpanzees (Pan troglodytes): implications for natural social systems and interindividual relationships. Am J Phys Anthropol 129(3):418-426. doi:10.1002/Ajpa.20289

Palagi E (2011) Playing at every age: modalities and potential functions in non-human primates. In: Pellegrini AD (ed) The Oxford handbook of the development of play. Oxford University Press, Oxford, pp 70-82

Palagi E, Antonacci D, Cordoni G (2007) Fine-tuning of social play in juvenile lowland gorillas (Gorilla gorilla gorilla). Dev Psychobiol 49(4):433-445. doi:10.1002/Dev.20219

Pellis S, Pellis V (2009) The playful brain: venturing to the limits of neuroscience. Oneworld Publications, Oxford

Poirier FE (1972) Primate socialization. Random House, New York

Poirier FE (1977) Introduction. Primate bio-social development: biological, social and ecological determinants. Garland, New York

Rapaport LG, Brown GR (2008) Social influences on foraging behavior in young primates: learning what, where, and how to eat. Evol Anthropol 17(4):189-201. doi:10.1002/evan.20180

Ron T, Mcgrew B (1988) Ecological assessment for a chimpanzee rehabilitation project in Northern Zambia. Primate Conserv 9:37-41

Russell JL, Lyn H, Schaeffer JA, Hopkins WD (2011) The role of socio-communicative rearing environments in the development of social and physical cognition in apes. Dev Sci 14(6):1459-1470. doi:10.1111/j.1467-7687.2011.01090.x

Russon AE (1990) The development of peer social interaction in infant chimpanzees: comparative social, Piagetian, and brain perspectives. Language and intelligence in monkeys and apes: comparative developmental perspectives. Cambridge University Press, Cambridge

Silk JB, Alberts SC, Altmann J (2003) Social bonds of female baboons enhance infant survival. Science 302(5648):1231-1234. doi:10.1126/science. 1088580

Spijkerman RP, Dienske H, vanHooff JARAM, Jens W (1996) Differences in variability, interactivity and skills in social play of young chimpanzees living in peer groups and in a large family zoo group. Behaviour 133:717-739. doi:10.1163/156853996x00440

Spinka M, Newberry RC, Bekoff M (2001) Mammalian play: training for the unexpected. Q Rev Biol 76(2):141-168. doi:10.1086/ 393866

Taborsky B, Oliveira RF (2012) Social competence: an evolutionary approach. Trends Ecol Evol 27(12):679-688. doi:10.1016/j.tree. 2012.09.003

Taborsky B, Arnold C, Junker J, Tschopp A (2012) The early social environment affects social competence in a cooperative breeder. Anim Behav 83(4):1067-1074. doi:10.1016/j.anbehav.2012.01. 037

van Hooff JARAM (1973) A structural analysis of the social behaviour of a semi captive group of chimpanzees. In: Cranach M, Vine I (eds) Social communication and movement: studies of interaction and expression in man and chimpanzee. Academic Press, London, pp 75-161

van Hooff JARAM (1972) A comparative approach to the phylogeny of laughter and smiling. In: Hinde RA (ed) Non-verbal communication. Cambridge University Press, Cambridge, pp 1-443

van Leeuwen EJC, Cronin KA, Haun DBM, Mundry R, Bodamer MD (2012) Neighbouring chimpanzee communities show different preferences in social grooming behaviour. Proc R Soc Lond B Biol Sci 279(1746):4362-4367. doi:10.1098/rspb.2012.1543

Vanschaik CP, Vanhooff J (1983) On the ultimate causes of primate social-systems. Behaviour 85:91-117. doi:10.1163/156853983x 00057

Watts DP, Pusey AE (2002) Behavior of juvenile and adolescent great apes. In: Pereira ME, Fairbanks LA (eds) Juvenile primates: life history, development, and behavior, 2nd edn. The University of Chicago Press, Chicago, pp 148-167

White DJ, Gersick AS, Freed-Brown G, Snyder-Mackler N (2010) The ontogeny of social skills: experimental increases in social complexity enhance reproductive success in adult cowbirds. Anim Behav 79(2):385-390. doi:10.1016/j.anbehav.2009.11.014 